(34.2\%), Cat (51.7\%), Dog (50.8\%) and cockroaches, Blatella germanica (48.3 8\%) and Periplaneta Americana (53.3\%). Overall, multiple sensitization (sensitization to $>4$ allergens) was significantly associated with increasing level of asthma severity $(\mathrm{p}<0.001)$. However, that association disappeared after adjustment for potential confounders like age, sex, exposure to smoking, pet and having family history of asthma or allergic rhinitis. It remained statistically significant only for the patients sensitized to HDM as the relationship was not modified by con founders. Moreover, mean SPT wheal size was not significantly associated with severity of asthma for all allergens tested.

Conclusions HDM was the most common sensitizing aeroallergen. Although children with moderate to severe asthma had significantly higher proportion of SPT positive, this study could not establish any relationship between degree of wheal size and severity of asthma. Therefore, the association between degree of sensitivity to indoor allergen and childhood asthma severity is still uncertain.

\section{DIAGNOSTIC DIFFICULTY DURING COVID 19 PANDEMIC- SICKLE CELL CRISIS OR PIMS-TS?}

Kyla Lief, Maggie Nyirenda. UK

10.1136/bmjpo-2021-RCPCH.65

Background The following case report highlights the difficulty in distinguishing Paediatric Multisystem Inflammatory Syndrome Temporally Associated with Sars- CoV- 2 (PIMS -TS) from sickle cell crisis. This is important in the context of a looming second wave of the Covid 19 pandemic, particularly in areas where there is a large population of children with sickle cell disease. This is the first case report discussing PIMS TS in a child with sickle cell disease and pulmonary emboli as a result of this.

Objectives A 17 year old boy with sickle cell disease presented to the paediatric department of a district general hospital in South London with pleuritic chest pain and worsening shortness of breath in April 2020. He was tachypnoeic and tachycardic. Oxygen saturation was $95 \%$ in air and he was afebrile. He had quiet breath sounds and was tender in the right upper quadrant of his abdomen. A week prior to this he required intravenous (IV) antibiotics and two exchange transfusions via a femoral line. Blood tests showed a white cell count (WCC) of 11.4 , a C-reactive protein (CRP) of 120, haemoglobin of 108 , INR of 1.6 and deranged liver function tests. His D dimer was 8339 , so a pulmonary Computed Tomography (CT) angiogram was performed. This demonstrated bilateral pulmonary emboli (PE).

Methods

Results Differential diagnoses for the cause of the PE were sickle cell disease and PIMS-TS.

Conclusions Both PIMS -TS and sickle cell crises are disorders involving exaggerated inflammation and risk of coagulopathy, with raised CRP, D Dimer, INR and WCC. The guidelines for investigations of PIMS TS include other markers such as LDH, troponin, BNP, ferritin and creatinine kinase. However, these blood tests are rarely performed in the general paediatric population or those with sickle cell disease, so they need to be specifically studied to determine whether they provide any significant distinction for a diagnosis of PIMS-TS.
In terms of pathophysiology, a diagnosis of $\mathrm{PE}$ as a complication of sickle cell is not surprising; however in clinical practice it is rarely seen. This points towards PIMS-TS being the cause of this presentation, for which WHO definition requires evidence of SARSCOV2 infection or exposure. Indeed our patient is likely to have been exposed to Covid 19, given that he presented in the peak of the first wave, however PCR swabs were negative during admission. Three months after presentation, his anti Sars Cov $2 \mathrm{IgG}$ was negative.

The key take home message from this case is to consider other diagnoses in sickle cell patients during the time of Covid 19. There is a need for increased research into how to differentiate the two disorders. This is important because if the primary cause of illness is PIMS-TS rather than sickle cell disease, careful consideration needs to go into treatments as immunomodulation with IV Ig may increase viscosity and steroids can contribute to hypertension, thus worsening the progression of underlying sickle cell disease. Diagnosing PIMS TS in those with sickle cell is particularly important as patients from a minority ethnic suffer worse outcomes.

\section{ENHANCING LEARNING AND IMPROVING FEEDBACK THROUGH QR CODES}

Kee Wei Phang, Poonam Patel. UK

\subsection{6/bmjpo-2021-RCPCH.66}

Background North Middlesex University Hospital is a District General Hospital based in Central London and has recently developed a weekly ED simulation programme as part of specialty training. The simulation programme is run by a simulation fellow and ED consultant and aims to improve trainees' confidence in core ED competencies including trauma and paediatric emergencies. It is recognised that there are difficulties in obtaining accurate and complete feedback from participants due to time constraints i.e. need to return to clinical work; because traditional methods of collecting feedback via paper forms are cumbersome and has an environmental impact with paper wastage, or feedback is lost through illegible writing. To improve the collection of feedback, we created a standardised online feedback form which participants access via a $\mathrm{QR}$ code using their mobile phones, and can be filled in quickly and accurately. This process will soon be rolled out to the entire hospital as the standardised method of collecting feedback from simulation sessions.

\section{Objectives}

1. To improve the collection of feedback after simulation sessions, such that the simulation team is better able to capture feedback from all participants and analyse a complete set of data.

2. To improve data analysis with electronic record by reducing human errors in transcribing feedback from paper forms during analysis.

3. To standardise the feedback collection process in the hospital.

4. Reduce paper wastage and become more environmentally friendly.

Methods Participants scan a QR code that is made available at the start and end of the simulation sessions which directs them to a short online feedback form where they can fill in feedback anonymously.

Results There is an average of $83 \%$ feedback response rate from the simulation sessions conducted between Sept 2020 to 
Dec 2020. There is no previous formal data on feedback collection prior to this. There is also a reduced use of paper for obtaining feedback as a result of this initiative, as no paper feedback forms were printed for simulation sessions. Participants who did not have a mobile phone or adequate internet access had the feedback forms sent via email or WhatsApp to them. Finally, the use of an QR code and online feedback tool resulted in accurate data analysis with no loss of data due to illegible writing and an ease of data analysis using Microsoft Excel.

Conclusions The use of QR codes has resulted in an improvement in feedback response rate from participants, as well as an improvement in the completeness and legibility of the data. We also note a high level of satisfaction from the participants in terms of the ease in providing feedback. As a team, we believe that the use of $\mathrm{QR}$ code to direct participants towards online feedback forms will be a sustainable and lasting method for the department. This will also improve the ability of the simulation team to continually improve delivery of the simulation sessions through accurate analysis of the feedback received.

\section{LOW FIDELITY IN SITU SIMULATIONS FOR SUPPORTING LEARNING}

Kee Wei Phang, Poonam Patel. UK

\subsection{6/bmipo-2021-RCPCH.67}

Background Simulation training has steadily been gaining traction in the medical training curriculum. Trainees are exposed to it from medical school in a simulation suite through to resuscitation courses such as the Advanced Paediatric Life Support. Majority of post graduate simulation training occurs in an artificial environment such as simulation suites which attempts to replicate the actual clinical environment with limited success. The simulation team in North Middlesex University Hospital Emergency Department (ED) identified a need for increased simulation training to improve trainee confidence in managing a range of cases presenting to the $\mathrm{ED}$, enhance inter-departmental team working and achieve core training competencies in a safe but realistic environment. In order to achieve this, a fully immersive simulation experience through low fidelity in situ simulation training is carried out in the department with participants performing their usual clinical roles.

Objectives

1. Provide a full immersive simulation experience for trainees, to enhance learning and improve clinical knowledge, skills and attitude.

2. Identification of latent errors within the working environment and the clinical processes.

3. Develop a more collaborative working relationship between ED and other departments through joint simulation exercises, fostering multidisciplinary teamwork.

Methods Low fidelity in situ simulation training is carried out weekly in the ED by the ED simulation faculty, usually comprising of the simulation fellow, a consultant and a senior staff nurse trained in providing feedback. Participants were identified at the start of the shift and given a pre-brief before commencement of the simulation. When the simulation concluded, the simulation faculty would facilitate the debrief, allowing for active discussion of any issues arising from the simulation. At the end of the session, feedback was then collected electronically on Survey Monkey and the data analysed to determine if the simulation session met its objectives.

\section{Results}

1. $93 \%$ of participants felt that it was relevant to their training

2. $94 \%$ of participants felt that it was a safe learning environment.

3. $85 \%$ of participants felt that they had achieved some clinical learning from the session.

4. Improved multidisciplinary team working was evident through the active participation of student nurses, nurses, doctors of different specialties in the sessions.

5. Identification of latent errors: It was noted from the simulation sessions that the anaesthetic team was unfamiliar with the anaesthetic equipment available to them in the new paediatric ED wing and work is currently being undertaken to support the anaesthetic team in familiarising with the new environment.

Conclusions Low fidelity in situ simulation training provides a cost effective, safe and realistic learning environment for trainees. It is also able to identify any latent errors within the clinical environment and/or pathways in the department and promotes a standardisation of practice because it was conducted in the actual clinical space. In addition, it fosters a more collaborative working relationship between departments who would otherwise not have the opportunity to develop this relationship. Therefore, through in situ stimulation trainings, it has a potential to improve clinical care and enhances patient safety; and should form an integral part of paediatric training.

\section{EFFECTIVENESS OF ORAL SEDATION FOR MRI IN YOUNG CHILDREN- EXPERIENCE IN A DISTRICT HOSPITAL}

Rajesh Balu, Sean Brennan. UK

\subsection{6/bmjpo-2021-RCPCH.68}

Background Elective MRI scans require a child to stay still, which is often achieved with either oral sedation or a general anaesthetic in a young child or those with cognitive or behavioural difficulties.

There is limited provision for use of general anaesthetic for elective MRIs within our DGH and majority of elective neuroimaging is done using oral sedation. The local protocol uses combination of Alimemazine Tartrate and Chloral Hydrate to achieve sedation in young children.

Objectives A service review was undertaken to assess effectiveness of oral sedation, complications and achievement of high quality MRI images.

Methods Elective appointments at paediatric day unit were evaluated retrospectively for 12 months from March 2019 to February 2020 (before the Covid-19 pandemic affected local services).

Data was analysed for 64 patients from electronic health records to look for effectiveness of sedation and outcomes.

Data was not evaluated for 8 children who were not brought for their appointment, 1 child who was not adequately fasted and 3 children who attended late so missed their MRI slot. 